\title{
Unresectable Ewing Sarcoma
}

National Cancer Institute

\section{Source}

National Cancer Institute. Unresectable Ewing Sarcoma. NCI Thesaurus. Code C132849.

A Ewing sarcoma which is not amenable to surgical resection. 